\title{
Focusing light with a reflection photon sieve
}

\author{
Matthias Kalläne, ${ }^{1, *}$ Jens Buck, ${ }^{1}$ Sönke Harm, ${ }^{1}$ Ralph Seemann, ${ }^{2}$ Kai Rossnagel,,${ }^{1}$ and Lutz Kipp ${ }^{1}$ \\ ${ }^{1}$ Institute of Experimental and Applied Physics, University of Kiel, D-24098 Kiel, Germany \\ ${ }^{2}$ Seemann \& Partner, D-20095 Hamburg, Germany \\ *Corresponding author: kallaene@physik.uni-kiel.de
}

Received March 8, 2011; revised May 13, 2011; accepted May 30, 2011;

posted May 31, 2011 (Doc. ID 143773); published June 20, 2011

\begin{abstract}
An advanced type of diffractive optical element is presented that combines the concept of the photon sieve with an offaxis, off-normal incidence reflection geometry. Compared to transmission optical elements, the signal-to-background ratio is significantly increased by separating the first from other diffraction orders without drastically reducing the size of the smallest diffractive element. The reflection photon sieve produces sharp foci at maximum contrast and offers the advantages of effective heat dissipation and a large working space above the focal plane. Experimental results for a device working at a photon energy of $100 \mathrm{eV}$ are presented and compared to theory. (c) 2011 Optical Society of America

OCIS codes: $230.1950,340.7480$.
\end{abstract}

The parallel progress in nanofabrication and in the generation of highly brilliant synchrotron radiation has significantly advanced many spectroscopy, scattering, and imaging techniques by pushing them to nanometer-scale spatial resolution over a large part of the electromagnetic spectrum [1,2]. In the extreme UV to soft x-ray regimes, where strong absorption rules out the use of refractive lenses []ㅡ, transmission Fresnel zone plates [3-6 $\underline{3}$ ] currently provide the best spatial resolution. Yet these diffractive optical elements commonly suffer from high background signals due to overlapping diffraction orders, strong secondary intensity maxima resulting from finite size effects, and limited working space between apertures and samples. Here we introduce a novel type of diffraction optics-a reflection photon sieve-that overcomes these limitations by using properly placed nanomirrors or nanoabsorbers [7] to obtain nanometer-scale foci of electromagnetic radiation in an off-axis, off-normal incidence reflection geometry. In addition to excellent focusing properties and good sample access, the design of the device allows for effective heat dissipation.

The diffractive imaging of a pointlike source by a reflection photon sieve is schematically illustrated in Fig. 1 . When monochromatic light illuminates the optical element under an angle of incidence $\alpha$, the wave is both directly reflected (zero-order diffraction) and focused into different spots under different diffraction angles $\beta$ (firstand higher-order diffraction). The curved dark areas on the device indicate the wave paths that contribute constructively to the focal spots. In the actual device these areas would be highly reflective, while the surrounding area would be made of a highly absorbing material. In coordinates of $r$ and $s$, the condition for constructive interference is given by

$$
r+s=p+q+m \cdot n \cdot \lambda,
$$

where $p$ and $q$ are the object and image distance, $m$ is the diffraction order, and $n \lambda$ the optical retardation between different diffraction areas ( $\lambda$, wavelength; $n$, integer). Their boundaries can be calculated by setting the optical retardation to $n \lambda \pm \frac{\lambda}{4}$. The optical element described so far is essentially a reflection zone plate [ $\underline{8}-\underline{10}]$. It becomes a reflection photon sieve by centering appropriately shaped nanomirrors, e.g., cylinders, on the dark areas such that the number of nanomirrors decreases smoothly from the center to the boundary of the illuminated area (see Fig. 1). This device thus combines the superior focusing properties of the photon sieve [7] with the advantages of an off-axis, off-normal incidence reflection geometry.

First, the array of nanomirrors can be designed such that a smooth window filter function is realized and secondary diffraction maxima are significantly suppressed [7].

Second, the off-axis illumination at off-normal incidence permits an effective filtering of unwanted diffraction orders, while at the same time providing access to the half-space above the focal plane and avoiding a significant decrease of the required minimum structure size of the optical element. For transmission Fresnel zone plates, it is well known that parasitic light, originating mainly from the unwanted diffraction orders, causes a high background signal in the focal plane [11]. A common solution is to use a central stop to block the directly transmitted light and to position an order-sorting aperture close to the focal plane to filter out the higher diffraction orders [Fig. 2(a)]. In contrast to this on-axis geometry, when illuminating zone plates off the optical axis $[\underline{12}, \underline{13}]$, off-axis central stops and order-sorting apertures are sufficient to block the parasitic light, thus

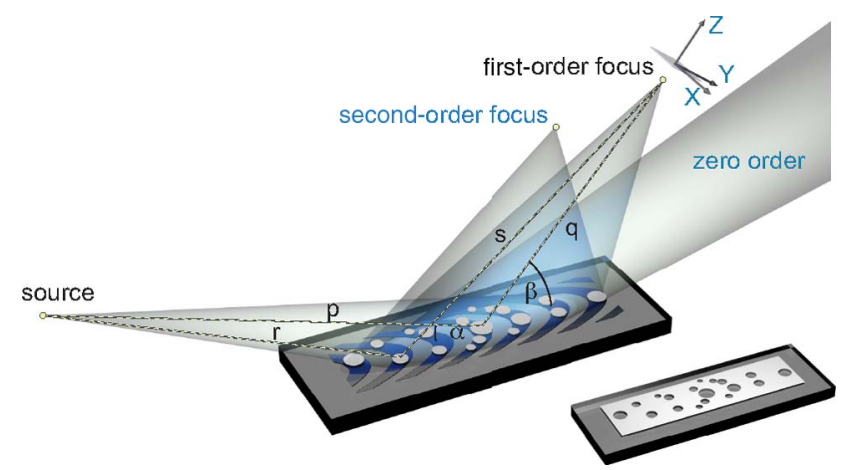

Fig. 1. (Color online) Point-to-point imaging with a reflection photon sieve. (center) Diffractive optical element consisting of appropriately shaped and arranged nanomirrors, each one centered on zones (dark rings) that lead to constructive interference. (bottom right) Corresponding inverted pattern of nanoabsorbers with the same diffraction properties. 
leaving one half above the focal plane easily accessible [Fig. 2(b)]. Note however that, for an optical element with a given minimum structure size, off-axis illumination leads to a reduction in the numerical (angular) aperture and thus also to a reduced spatial resolution [compare the aperture angles $\gamma_{1}$ and the intensity profiles in the focal planes in Figs. 2(a) and 2(b)]. Yet, remarkably, this effect can partly be compensated for by choosing an off-normal angle of incidence $\left(\alpha \neq 90^{\circ}\right)$. Figure 3 shows the theoretical minimum structure size of a reflection zone plate that is required for a spatial resolution of $\Delta x=$ $\Delta y=200 \mathrm{~nm}$ (FWHM). For each incidence and diffraction angle pair $(\alpha, \beta)$, the corresponding device geometry is calculated using Eq. (1) before the smallest structure size is determined ( $p=1 \mathrm{~m}, q=20 \mathrm{~mm}, h \nu=100 \mathrm{eV})$. In the resulting image, the broadening of the "diagonal" intensity distribution toward lower angles is the key. Consider, e.g., an angle separation of $|\alpha-\beta|=3^{\circ}$. In the case of normal incidence $\left(\alpha=90^{\circ}\right)$, the required minimum structure size is $w_{\min } \approx 74 \mathrm{~nm}$ [configuration (a) of Fig. 3]. For $\alpha=21^{\circ}$, on the other hand, the required structure size is about twice as large and only $\sim 20 \%$ smaller than the spatial resolution: $w_{\text {min }} \approx 158 \mathrm{~nm}$ [configuration (b) of Fig. 3].

Third, the implementation of a reflection geometry helps to solve two practical problems connected with the use of transmission optics. On one hand, the reflection geometry is an obvious solution to break the general resolution limit of transmission optics due to volume diffraction effects [14], in the extreme UV and soft x-ray regimes $[\underline{8}-10,15]$ as well as for hard $x$ rays $[\underline{16}-19]$. On the other hand, to prevent radiation damage, a reflection optical element can be simply and effectively cooled. Note that heat dissipation on the optical element is even enhanced when the sieve structure is inverted (Babinet's principle) because the absorbing area becomes significantly smaller (see Fig. 1).

To verify the focusing properties of reflection photon sieves, we have performed numerical simulations and first measurements with a real device. The expected focal intensity profiles were calculated along the $x, y$, and $z$ directions toward the zero-order light (see Fig. 1) using Fresnel-Kirchhoff diffraction theory. The basic parameters for all considered diffraction optics are $p=1 \mathrm{~m}$, $q=20 \mathrm{~mm}, h \nu=100 \mathrm{eV}$, and $\Delta x=\Delta y \approx 300 \mathrm{~nm}$ (FWHM of the focal spot). The reflection devices were designed for $\alpha=21^{\circ}$ and $\beta=24^{\circ}$, and for the reflection photon sieve a Weber-type transmission window was implemen- (a)

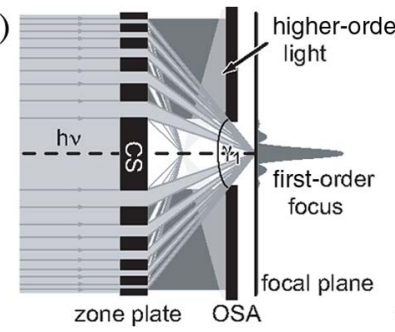

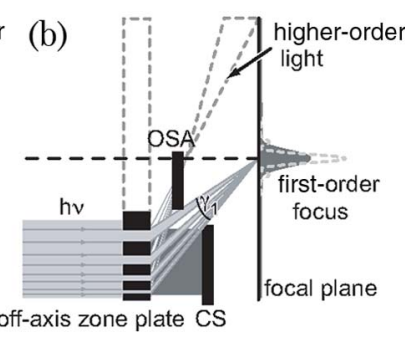

(b)
Fig. 2. (Color online) Separation of first- and zero-order light for conventional transmission Fresnel zone plates. (a) On-axis and (b) off-axis illumination. In both cases a central stop (CS) and an order-sorting aperture (OSA) are used. Typical intensity profiles in the focal plane are indicated.

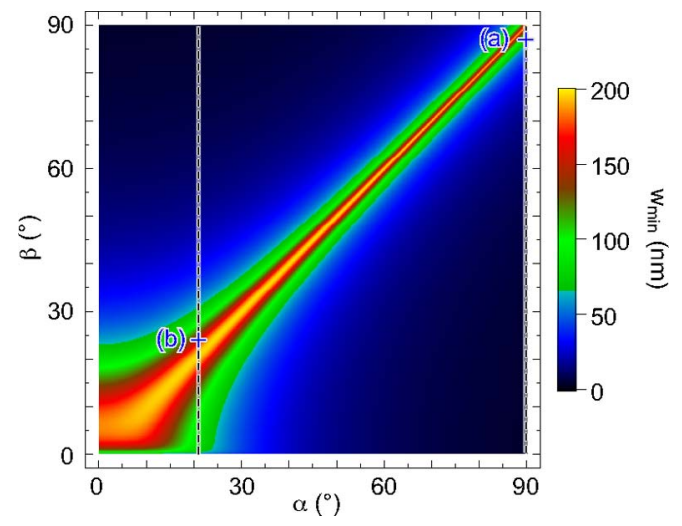

Fig. 3. (Color online) Smallest structure size $\left(w_{\min }\right)$ of a diffractive optical element, as required to achieve a spatial resolution of $\Delta x=\Delta y=200 \mathrm{~nm}$. False color representation of the dependence of $w_{\min }$ on the angle of incidence $\alpha$ and the diffraction angle $\beta$. Two configurations for $|\alpha-\beta|=3^{\circ}$ are indicated: (a) $\alpha=90^{\circ}$ and (b) $\alpha=21^{\circ}$.

ted [7]. The real test device is an inverted reflection photon sieve, manufactured by electron beam lithography, i.e., by etching polygon-shaped nanoabsorbers in an $80 \mathrm{~nm}$ thick chromium layer on a quartz glass substrate. The device is optimized for third-order diffraction and should allow for a nominal spatial resolution of $\Delta x=$ $\Delta y \approx 300 \mathrm{~nm}$ at a minimum structure size of $\sim 475 \mathrm{~nm}$ (device dimensions, semimajor axis $\approx 1.5 \mathrm{~mm}$, semiminor axis $\approx 0.6 \mathrm{~mm}, 1669$ equivalent Fresnel zones contributing to the focal spot). In the experiments, the reflection photon sieve was used to image a pinhole aperture of $20 \mu \mathrm{m}$ diameter onto the focal plane so that the expected total spatial resolution is $\Delta x=\Delta y \approx 400 \mathrm{~nm}$. The focal spot profiles were characterized by scanning a sharp knife edge across the focus in the $y$ direction and measuring the intensity of the transmitted light. All measurements were carried out with $100 \mathrm{eV}$ photons at the undulator beamline Bypass Wiggler 3 of the Hamburg Synchrotron Radiation Laboratory.

Figure 4(a) compares the calculated focal intensity profiles of a transmission Fresnel zone plate, a reflection zone plate, and a reflection photon sieve in the $y$ direction (the focal profile in the $x$ direction is similar and not shown here). For small $y$ values near the focal maximum, the three intensity profiles are almost identical. At larger distances $(y>0.3 \mu \mathrm{m})$, however, significant deviations occur due to the different window functions and geometries of the optical devices. For the transmission zone plate, the overlap of diffraction orders and secondary intensity maxima lead to a high background intensity. For the reflection zone plate, the background signal is already lowered due to the effective separation of diffraction orders. And yet, for the reflection photon sieve, the use of the smooth window function reduces the background level by about another 4 orders of magnitude, making the reflection photon sieve the device providing the highest contrast.

Figures 4(b) and 4(c) show the experimental results obtained with the test device. The derivative of the knife-edge scanned intensity distribution represents a one-dimensional projection of the two-dimensional spot profile and provides a measure for the focal spot width [Fig. 4(b)]. Such projections are plotted in Fig. $\underline{4(\mathrm{c})}$ as a 

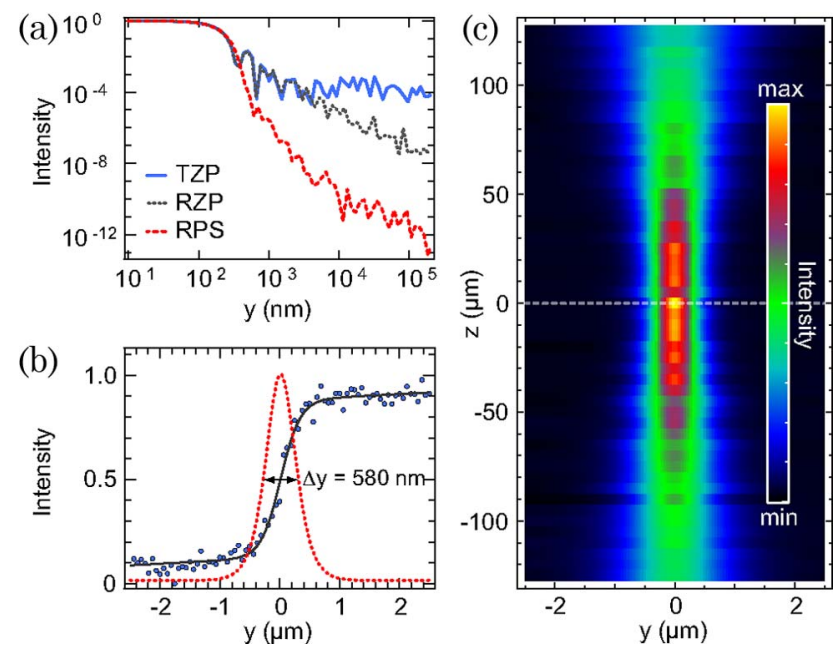

Fig. 4. (Color online) Focal plane intensities of various diffraction optics with a spatial resolution of $\Delta x=\Delta y \approx 300 \mathrm{~nm}$. (a) Calculated focal plane intensities for a transmission Fresnel zone plate (TZP, blue solid curve), a reflection zone plate (RZP, gray dotted curve), and a reflection photon sieve with Webertype transmission window (RPS, red dashed curve). (b) Focal intensity distribution of a prototype reflection photon sieve (blue dots), measured by a knife-edge scan. The spatial resolution is given by the FWHM of the derivative (red dashed curve) of the fit (gray solid curve) to the experimental data. (c) Intensity derivative as a function of $z$.

function of $z$, i.e., the distance between focus and knife edge. The sharpest intensity profile at $z=0$ has a FWHM of $\Delta y \approx(580 \pm 60) \mathrm{nm}$ [Fig. 4(b)]. This value and also the extracted value for the depth of focus $(\Delta z \approx 50 \mu \mathrm{m})$ agree with the design values within the uncertainties of the experiment. In addition, we could confirm a clear separation of the focal spot and the zero-order light of about $500 \mu \mathrm{m}$ (not shown). We may thus conclude that the measured performance of the prototype reflection photon sieve demonstrates the functioning of the concept.

In view of the broad availability of third-generation synchrotron radiation, we expect that reflection photon sieves will be successfully applied in synchrotron-based techniques that require nanometer-scale foci, high contrast, and good sample access at medium efficiency. Moreover, because of the expected radiation hardness, these devices could be used as focusing elements for freeelectron laser radiation. Depending on materials properties, reflection photon sieves should be applicable over a large part of the electromagnetic spectrum. Possible limitations due to the reduced reflectivities toward higher photon energies and lower incidence angles could partly be compensated for by using multilayer mirrors or total external reflection, yet at the expense of a restricted angle range for such more advanced devices.

\section{References}

1. M. Kiskinova, M. Marsi, E. di Fabrizio, and M. Gentili, Surf. Rev. Lett. 6, 265 (1999).

2. A. Sakdinawat and D. Attwood, Nat. Photon. 4, 840 (2010).

3. G. Schmahl, D. Rudolph, P. Guttman, and O. Christ, Zone Plates for X-Ray Microscopy (Academic, 1984).

4. W. Chao, B. H. Harteneck, J. A. Liddle, E. H. Anderson, and D. T. Attwood, Nature 435, 1210 (2005).

5. J. Vila-Comamala, K. Jefimovs, J. Raabe, T. Pilvi, R. H. Fink, M. Senoner, A. Maassdorf, M. Ritala, and C. David, Ultramicroscopy 109, 1360 (2009).

6. W. Chao, J. Kim, S. Rekawa, P. Fischer, and E. H. Anderson, Opt. Express 17, 17669 (2009).

7. L. Kipp, M. Skibowski, R. L. Johnson, R. Bernd, R. Adelung, S. Harm, and R. Seemann, Nature 414, 184 (2001).

8. T. Wilhein, D. Hambach, B. Niemann, M. Berglund, L. Rymell, and H. M. Hertz, Appl. Phys. Lett. 71, 190 (1997).

9. T. Wilhein, D. Altenbernd, U. Teubner, E. Förster, R. Hässner, W. Theobald, and R. Sauerbrey, J. Opt. Soc. Am. B 15, 1235 (1998).

10. A. G. Michette, S. J. Pfauntsch, A. Erko, A. Firsov, and A. Svintsov, Opt. Commun. 245, 249 (2005).

11. D. Attwood, Soft X-Rays and Extreme Ultraviolet Radiation (Cambridge University, 1999).

12. B. Niemann, P. Guttmann, D. Hambach, G. Schneider, D. Weiss, and G. Schmahl, Nucl. Instrum. Methods Phys. Res. A 467-468, 857 (2001).

13. D. Hambach, M. Peuker, and G. Schneider, Nucl. Instrum. Methods Phys. Res. A 467-468, 877 (2001).

14. C. Bergemann, H. Keymeulen, and J. E. van der Veen, Phys. Rev. Lett. 91, 204801 (2003).

15. B. Niemann, T. Wilhein, T. Schliebe, R. Plontke, O. Fortagne, I. Stolberg, and M. Zierbock, Microelectron. Eng. 30, 49 (1996).

16. C. G. Schroer and B. Lengeler, Phys. Rev. Lett. 94, 054802 (2005).

17. C. Fuhse, C. Ollinger, and T. Salditt, Phys. Rev. Lett. 97, 254801 (2006).

18. F. Pfeiffer, C. David, J. F. van der Veen, and C. Bergemann, Phys. Rev. B 73, 245331 (2006).

19. H. C. Kang, J. Maser, G. B. Stephenson, C. Liu, R. Conley, A. T. Macrander, and S. Vogt, Phys. Rev. Lett. 96, 127401 (2006). 